\title{
自由基的热力学和动力学定量参数
}

\author{
黄谋新 $\dagger$ 贾宗宾†, $, c, c$ 罗三中 $*, a, b, c$ 程津培*,a,d \\ ( ${ }^{a}$ 清华大学化学系 基础分子科学中心 北京 100084) \\ ( $b$ 中国科学院化学研究所 分子识别与功能重点实验室 北京 100190) \\ ( ${ }^{c}$ 中国科学院大学 北京 100490) \\ ( $d$ 南开大学化学学院 元素有机化学国家重点实验室 天津 300071)
}

\begin{abstract}
摘要 近十余年来, 自由基化学重新焕发了生机，在合成化学和材料科学等领域取得了突破性的进展. 与突飞猛进的 合成方法学研究相比，人们对自由基反应的机制以及规律的研究和总结相对滞后. 另一方面，自由基一直是物理有机 化学领域重要的研究对象, 在横跨一个世纪的研究历程中积累了大量热力学和动力学参数, 但这些数据大多数都淹没 在文献中, 缺乏系统的汇编和整理, 从而不被大多数合成化学家所熟知. 本综述拟对自由基热力学和动力学定量参数 进行系统的汇编和整理, 以期为相关领域的理性设计与发展提供支撑数据. 这些数据参数主要包括自由基稳定化能 $(\mathrm{RSE}) 、$ 自由基寿命 $(\tau)$ 、取代基常数 $(\sigma)$ 、亲电性指数 $(\omega)$ 等. 以这些数据为基础, 将梳理一些重要的自由基化学基本概 念如自由基稳定性、Class S/O 自由基取代基效应、稳态和瞬态自由基效应等
\end{abstract}

关键词 自由基性质; 定量参数; 稳定性; 取代基效应; 稳态自由基效应

\section{Quantitative Thermodynamic and Kinetic Parameters of Radical}

\author{
Huang, Mouxin ${ }^{\dagger, a} \quad$ Jia, Zongbin ${ }^{\dagger, b, c} \quad$ Luo, Sanzhong ${ }^{*, a, b, c} \quad$ Cheng, Jin-Pei ${ }^{*, a, d}$ \\ ( ${ }^{a}$ Center for Basic Molecular Science, Department of Chemistry, Tsinghua University, Beijing 100084) \\ $\left({ }^{b}\right.$ Key Laboratory of Molecular Recognition and Function, Institute of Chemistry, \\ Chinese Academy of Sciences, Beijing 100190) \\ ( ${ }^{c}$ University of Chinese Academy of Sciences, Beijing 100490) \\ ( ${ }^{d}$ State Key Laboratory of Elemento-organic Chemistry, College of Chemistry, Nankai University, Tianjin 300071)
}

\begin{abstract}
Radical chemistry has gained its renaissence in the past decade and trendendous progresses have been witnessed in synthetic and material chemistry. However, mechanism studies are largely lagging behind comparing with rapid paces in the development of synthetic methodologies. On the other hand, the study of radical species remains a central theme in physial organic chemistry, and a large amount of thermodynamic and kinetic data on radicals have been generated over a century's reserch. Unfortuately, there has been no systematic compilation and curation of these quatitative data that are dispersedly distributed and buried in literature. As a result, most synthetic chemists are not acquainted with these valuable data. In this review, we aim in compiling and curating thermodynamic and kinetic parameters of radicals, that may hopefully provide a quantitative data basis for rational development and evolution of radical chemistry. The key parameters include radical stability energy (RSE), radical lifetime $(\tau)$, substituent constant $(\sigma)$ and electrophilic index $(\omega)$. Fundamental concepts such as radical stability, Class S/O radical substitution effect, persistent/transient radical effect will be discussed on the basis of data.
\end{abstract}

Keywords radical property; quantitative parameters; stability; substituent effect; persistent radical effect

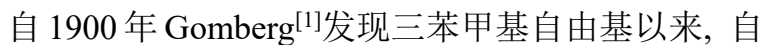
由基就引起了化学家的广泛关注, 但其活泼的反应性质 为自由基的深入研究带来了挑战, 自由基反应一度被认
为是混乱且不可控的 ${ }^{[2]}$. 随着有机化学和现代仪器手段 的飞速发展，自由基的神秘面纱被缓缓揭开，一大批基 于自由基中间体的化学反应相继涌现 ${ }^{[3]}$, 使自由基在药

\footnotetext{
* Corresponding authors. E-mail: luosz@tsinghua.edu.cn; jinpei_cheng@tsinghua.edu.cn

Received June 8, 2021; revised June 27, 2021; published online July 12, 2021

Dedicated to the 100th anniversary of Chemistry at Nankai University.

Project supported by the National Natural Science Foundation of China (Nos. 21672217, 21861132003, 22031006).

国家自然科学基金(Nos. 21672217, 21861132003, 22031006)资助项目.

†共同第一作者(These authors contributed equally to this work).
} 
物研发 ${ }^{[4]}$ 、农业化学 ${ }^{[5]}$ 、材料科学 ${ }^{[6]}$ 等领域发挥着不可替 代的作用. 然而, 人们对自由基性质以及反应机理、规 律的认识大多来自于对反应结果的定性分析, 适用性和 准确性不足, 一定程度上阻碍了自由基化学的发展. 一 直以来, 物理有机化学家致力于自由基反应机理的研 究, 积累了大量热力学、动力学数据. 这些定量化指标 是人们理解自由基性质, 进而理性设计新方法新反应的 重要依据. 但这些数据大多数淹没在文献中, 尚缺乏系 统的汇编和整理, 因而未能得到广泛的应用. 本文将系 统整理有关自由基的定量化参数, 该方向的研究时间跨 度大, 内容繁杂. 综述将重点从影响自由基活性和反应 性的热力学、动力学和极性匹配效应等三个方面遴选定 量化参数.

\section{1 自由基的热力学稳定性}

作为一种活性中间体, 自由基的稳定性可从热力学 和动力学两个方面来衡量. 自由基热力学稳定性以研究 自由基自身在热力学状态下的本征稳定性为目的, 通常 使用化学键的均裂键能(BDE, bond dissociation energy) 来表征 ${ }^{77}$. BDE 提供了有关键强度的重要信息, 是自由 基热力学的重要基本参数 ${ }^{[8]}$, 可由 $\mathrm{Eq} .1$ 的反应焓测定. 一般讲, BDE 越小, 表示生成的自由基就越稳定, 反之 则越不稳定. 以往的 BDE 测量方法包括热解法、卤素自 由基动力学法、光电离质谱法和负离子循环法等 ${ }^{[8]}$. 这 些气相测量方法不但十分繁琐, 而且产生的误差相比于 $\mathrm{p} K_{\mathrm{a}}$ 测定也大许多 $\left(12 \sim 16 \mathrm{~kJ} \cdot \mathrm{mol}^{-1}\right)$. 此外，这些策略对 底物限制性也比较大, 对于大多数有机化合物不太适 用.

$$
\begin{aligned}
\mathrm{R} & -\mathrm{H} \rightarrow \mathrm{R}^{\cdot}+\mathrm{H}^{\cdot} \\
\mathrm{BDE} & =\Delta H_{f 298}^{0} \\
& =\Delta_{f} H^{0}\left(\mathrm{R}^{\cdot}\right)+\Delta_{f} H^{0}\left(\mathrm{H}^{\cdot}\right)-\Delta_{f} H^{0}(\mathrm{R}-\mathrm{H})
\end{aligned}
$$

1988 年, Bordwell 和程津培等 ${ }^{[9]}$ 利用有机化合物氧 化还原电位和酸性解离常数, 建立了一套通过热力学循 环来估算溶液中 $\mathrm{C}-\mathrm{H}$ 键 BDE 的方法(图 1). 该方法简 单易操作, 只需测量 $\mathrm{p} K_{\mathrm{HA}}$ 和 $E_{\mathrm{ox}}\left(\mathrm{A}^{-}\right)$即可通过 $\mathrm{Eq} .3$ 求得 BDE. 式中所用的 $\mathrm{p} K_{\mathrm{HA}}$ 可用指示剂重叠法得到, $E_{\mathrm{Ox}}\left(\mathrm{A}^{-}\right)$ 可用循环伏安法测得. $C$ 是包含 $E_{\mathrm{rd}}\left(\mathrm{H}^{+}\right)$在内的各项常数 之和, 其值在二甲基亚砜(DMSO)中为 $233.6 \mathrm{~kJ} \cdot \mathrm{mol}^{-1}$. 该方法精度优于各类气态化学方法, 效率高, 适用范围 广泛，原则上适用于所有可测量 $\mathrm{p} K_{\mathrm{a}}$ 的分子，也可以方 便地扩展到其他溶剂体系. 此外, 对于气相方法测定困 难的较大分子, 该方法更为适用.

$$
\mathrm{BDE}=5.507 \mathrm{p} K_{\mathrm{HA}}+96.49 E_{\mathrm{ox}}\left(\mathrm{A}^{-}\right)+C
$$

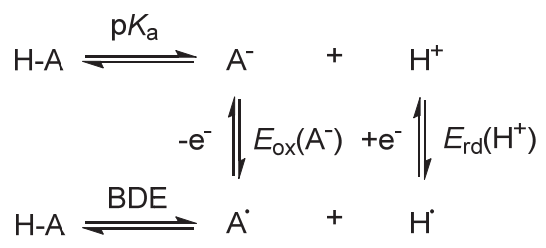

图 1 推导 $\mathrm{BDE}$ 的热力学循环示意图

Figure 1 Thermodynamic cycle diagram for deriving BDE

Bordwell 和程津培基于该公式测定了一系列的苄

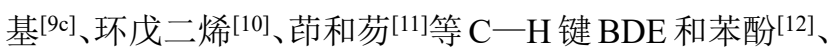
硫酚 $\left[9 \mathrm{c}\right.$ 和苯胺 ${ }^{[13]}$ 等杂原子 $\mathrm{X}-\mathrm{H}$ 键的 $\mathrm{BDE}$, 测定结果与 先前报道的气相测量法十分吻合，证实了该策略的准确 性. 此外, 利用该方法还可以很方便地测定苯乙腈[14]、 苯磺酰胺 ${ }^{[15]}$ 等传统气相方法不能适用的底物 ${ }^{[16]}$, 随后 基于此测定了一系列化合物 BDE 的数据 ${ }^{[17]}$.

自由基属于缺电子体系，一般来讲，供电子基 (EDG, electron-donating group)可以使其趋于稳定, 而吸 电子基(EWG, electron-withdrawing group)对自由基的影 响比较复杂, 一方面可以通过对单电子的共振离域作用 使其稳定化，另一方面，其诱导效应又可以使自由基更 缺电子, 起到去稳定化的作用 ${ }^{[18]}$. 早在 1966 年, Walter ${ }^{[19}$ 就发现 $p-\mathrm{NO}_{2}$ 取代基可使平衡 I 右移, 但使平 衡 II 左移(图 2a). 据此观察, 他将自由基分为两类: 一 类为 Class $\mathrm{S}$ 自由基( $\mathrm{S}$ 表示 same), 其供电子基和吸电子 基对自由基稳定化的作用方向相同; 另一类为 Class $\mathrm{O}$ 自由基( $\mathrm{O}$ 表示 opposite), EDG 和 EWG 对自由基稳定化 作用相反. Walter 认为, Class $\mathrm{S}$ 自由基的特点是自由基 中心原子上只有一个单电子, 如甲基自由基、芐基自由 基等. 而自由基中心除含有单电子外还同时具有孤对电 子的自由基则为 Class $\mathrm{O}$ 自由基, 如 $\mathrm{N}$ 自由基(图 2b). 虽 然没有新的实验证据，他还预测 $\mathrm{O} 、 \mathrm{~S}$ 等杂原子自由基 应该也属于 Class $\mathrm{O}$ 类, 但一直未得到实验证实. 其后 虽有许多有关自由基热力学稳定性的取代基效应的研

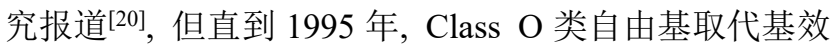
应才通过程津培课题组对杂原子自由基的系统研究被 证实普遍存在 ${ }^{[18,21]}$. 该研究指出, 由于 $\mathrm{EWG}$ 可能会与 自由基中心的孤对电子发生作用, 进而造成该原子的单 电子趋于定域, 因此导致自由基的稳定性降低. 如图 $2 \mathrm{c}$, 以苯酚自由基为例[21b], 相对于未取代的苯酚, 对硝 基苯酚自由基中心的孤对电子更易于处于 $\mathrm{p}$ 轨道并与苯 环离域, 使得自由基定域于 $\sigma$ 轨道, 此时的取代基不但 不能使之离域, 反而会因拉电子诱导效应进一步突出了 自由基的缺电子性质, 故其稳定性降低.

程津培等对一系列的底物的 BDE 进行了测试, 通 过对自由基稳定化能(RSE, radical-stabilizing energy, 即 
(a) Walter's opinion

$$
\begin{aligned}
& \mathrm{Ar}_{3} \mathrm{C}-\mathrm{CAr}_{3} \rightleftharpoons 2 \cdot \mathrm{CAr}_{3} \\
& \mathrm{Ar}_{2} \mathrm{~N}-\mathrm{NAr}_{2} \rightleftharpoons 2 \cdot \mathrm{NAr}_{2}
\end{aligned}
$$

(b) Classification of Class S/O radical

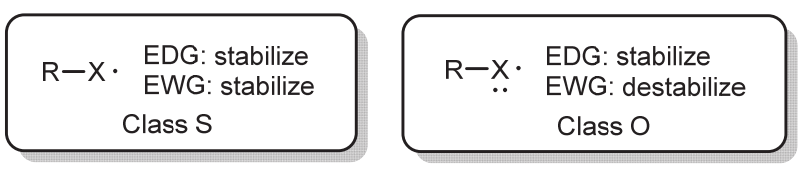

(c) Resonance structure of $p$-nitrophenoxyl radical

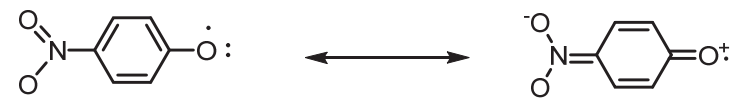

图 2 Class $\mathrm{S}$ 自由基与 Class $\mathrm{O}$ 自由基的结构特征

Figure 2 Resonance structure of $p$-nitrophenoxyl radical

取代底物与未取代底物 BDE 的差值)比较后发现, 简单 的烷基自由基, 如甲基自由基、芐基自由基等的确都属 于 Class S 自由基. 但随后他们研究发现, 碳自由基并不 全是 Class $\mathrm{S}$ 自由基. 程津培组通过测定一系列苯乙腈 衍生物的键均裂能(BDE) 数据发现 ${ }^{[21 a]}$, 芐位的氢被吸电 子的氰基取代后, $\mathrm{C}-\mathrm{H}$ 键的 $\mathrm{p} K_{\mathrm{a}}$ 和 $\mathrm{BDE}$ 相对于甲苯 $\left(\mathrm{BDE}=368.2 \mathrm{~kJ} \cdot \mathrm{mol}^{-1}\right)$ 均有一定降低 $(\mathrm{BDE}=343.4$ $\left.\mathrm{kJ} \cdot \mathrm{mol}^{-1}\right)$ (表 1). 进而发现, 此时该自由基已表现出了一 定的 Class $\mathrm{O}$ 自由基的性质. 当第二个吸电子基团酯基 引入后, 芐位 $\mathrm{C}-\mathrm{H}$ 键的 $\mathrm{p} K_{\mathrm{a}}$ 和 $\mathrm{BDE}$ 进一步降低, 该自 由基表现出更显著的 Class $\mathrm{O}$ 性质. 结合以往文献报道, 不论是动力学角度还是热力学角度的研究, 都显示出通 常苠基自由基属于 Class $\mathrm{S}$ 自由基的情况 ${ }^{[7,22]}$. 程津培认 为, 对自由基的性质起决定性作用的应该是自由基中心 原子的表观电负性而非未成对电子. 这一结论在对一系 列具有高电负性的亚甲基 $\mathrm{C}-\mathrm{H}$ 键键能考察后得到了验 证 ${ }^{[23]}$. 即对于原本呈现 Class S 性质的自由基, 通过吸电
子基的引入，可以转变为 Class $\mathrm{O}$ 性质的自由基.

随后，程津培等 ${ }^{[2-13,24]}$ 通过对一系列取代的苯酚、 苯硫酚和苯胺类底物的 $\mathrm{X}-\mathrm{H}$ 键 $\mathrm{BDE}$ 的测定, 确定它们 都属于 Class $\mathrm{O}$ 自由基. 对于苯胺类底物, 当氮原子上 一个氢被乙酰基取代后 ${ }^{[21 a]}, \mathrm{N}-\mathrm{H}$ 键的键能增加, 使得 氮自由基去稳定, 这与其对碳自由基的影响正好相反. 此时苯环对位的 EWG 同样会引起氮自由基热力学稳定 性降低, 但是由于饱和效应，其数值并没有苯胺的大 (表 1, RSE = radical-stabilizing energy). 通过对 EDG 的 比较可以发现, 由于乙酰基苯胺更缺电子，同一供电子 基对乙酰基苯胺自由基的稳定化程度要远大于苯胺 ${ }^{[25]}$. 相对于乙酰基苯胺, 由于取代基与中心原子的作用距离 增加，苯乙酮缩胺脲的取代基效应则明显弱化 ${ }^{[26]} .1998$ 年, 程津培等进一步系统地研究了氮上的双取代基对其 自由基热力学稳定性的影响 ${ }^{[18]}$. 对于双芳基取代的氮 自由基, 第二个芳环的引入产生的稳定化能(19 kJ・ $\left.\mathrm{mol}^{-1}\right)$ 远小于第一个芳环带来的影响 $\left(63 \mathrm{~kJ} \cdot \mathrm{mol}^{-1}\right)$. 当 双取代基之一为具有强供电子能力的杂原子时(如 $\mathrm{OH}$, $\mathrm{NH}_{2}$ 等), 取代基可与自由基中心形成 “两中心三电子” 键来提高自由基的稳定性. 对于 EWG 基团, 则有两种 截然相反的结果. 膦酰基中由于 $\mathrm{P}=\mathrm{O}$ 键的反馈 $(\mathrm{p}-\mathrm{d}) \pi$ 键可以使氮上的单电子离域，进而贡献一部分稳定化作 用，表现出比乙酰基稍弱的去稳定化作用. 但吸电子能 力更强的 $\mathrm{Ph}_{3} \mathrm{P}^{+}$取代基通过形成 $\mathrm{P}=\mathrm{N}$ 双键 “离域通道” 让单电子离域, 进而大幅稳定氮自由基. 后续的理论计 算工作也对上述规律进行了验证 ${ }^{[27]}$.

综合以上分析可以发现，不论是碳自由基还是杂原 子自由基, 随着吸电子基团的引入, 自由基中心的表观 电负性逐渐增强, 其 Class O 属性随之也更加明显. 在 这一过程中, 烷基 $\mathrm{C}-\mathrm{H}$ 键的 $\mathrm{BDE}$ 和 $\mathrm{p} K_{\mathrm{a}}$ 也随之降低, 趋势一致. 杂原子 $\mathrm{N}-\mathrm{H}$ 键在这一过程中 $\mathrm{p} K_{\mathrm{a}}$ 逐渐降低 的同时, $\mathrm{BDE}$ 却逐渐升高, 二者趋势相反. 对于连有供

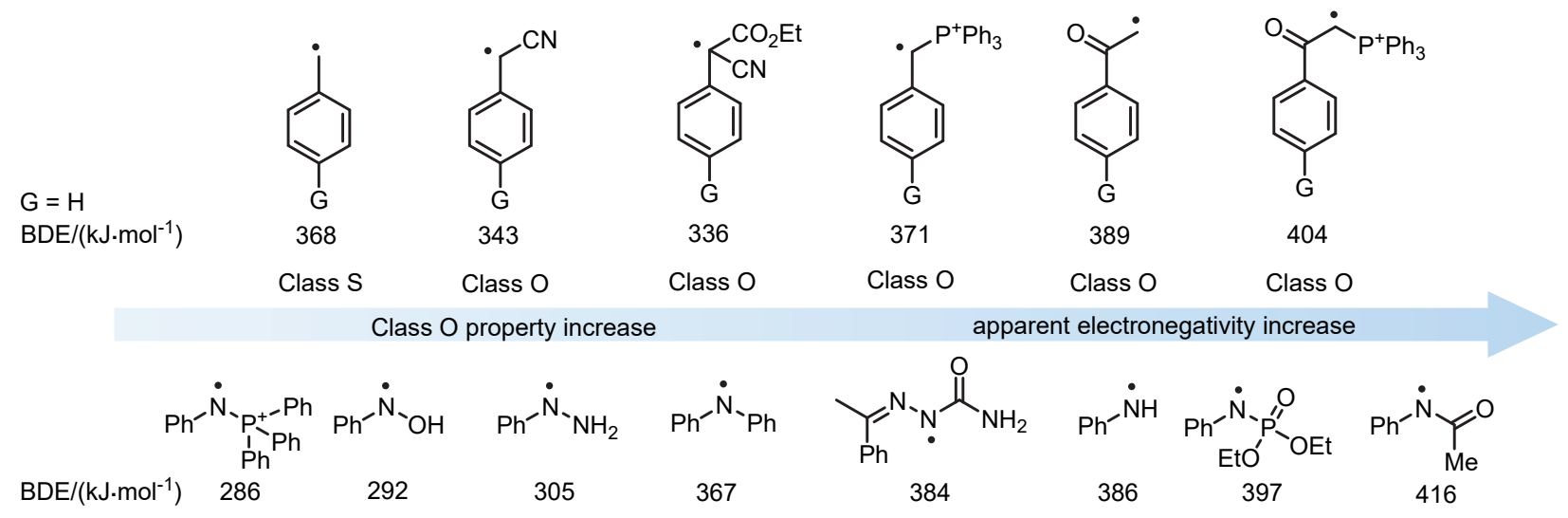

图 3 碳自由基和氮自由基的取代基效应

Figure 3 Substituent effects of carbon and nitrogen radicals 
表 1 部分 $\mathrm{C}-\mathrm{H}$ 和 $\mathrm{N}-\mathrm{H}$ 键均裂能和自由基稳定化 能 ${ }^{[18,21 \mathrm{a}, 23 \mathrm{a}, 26-27]}$

Table 1 BDEs and RSEs of selected $\mathrm{C}-\mathrm{H}$ and $\mathrm{N}-\mathrm{H}$ bonds

\begin{tabular}{|c|c|c|c|c|c|c|}
\hline \multirow{2}{*}{ Substituent } & \multicolumn{2}{|c|}{$\mathrm{ArCH}_{3}$} & \multicolumn{2}{|c|}{$\mathrm{ArCH}_{2} \mathrm{CN}$} & \multicolumn{2}{|c|}{$\mathrm{ArCH}(\mathrm{CN}) \mathrm{CO}_{2} \mathrm{Et}$} \\
\hline & $\mathrm{BDE}$ & $\mathrm{RSE}^{a}$ & $\mathrm{BDE}$ & RSE & $\mathrm{BDE}$ & RSE \\
\hline 4-OMe & 362.2 & 6.0 & 337.1 & 6.3 & 327.9 & 8.4 \\
\hline 4-Me & 366.6 & 1.6 & 340.9 & 2.5 & 334.8 & 1.5 \\
\hline $\mathrm{H}$ & 368.2 & (0) & 343.4 & $(0)$ & 336.3 & (0) \\
\hline $4-\mathrm{CO}_{2} \mathrm{Me}$ & - & - & 346.6 & -3.2 & 343.2 & -6.9 \\
\hline 4-CN & 388.0 & 0.2 & 345.4 & -2.0 & 348.1 & -11.8 \\
\hline \multirow{2}{*}{$\begin{array}{c}\text { Substituent } \\
\left(\mathrm{E}^{+}\right)\end{array}$} & \multicolumn{2}{|c|}{$\mathrm{Ph}-\mathrm{CH}_{2}-\mathrm{E}^{+}$} & \multicolumn{2}{|c|}{$\mathrm{Ph}-\mathrm{COCH}_{2}-\mathrm{E}^{+}$} & \multicolumn{2}{|c|}{ 9-E $\mathrm{E}^{+}$-fluorene } \\
\hline & $\mathrm{BDE}$ & RSE & BDE & RSE & $\mathrm{BDE}$ & RSE \\
\hline $\mathrm{H}$ & 370.5 & (0) & 388.9 & (0) & 332.5 & (0) \\
\hline $\mathrm{Ph}_{3} \mathrm{P}^{+}$ & 370.9 & -0.4 & 403.6 & -14.6 & 341.3 & -8.8 \\
\hline$\left.{ }^{\imath-\mathrm{Bu}}\right)_{3} \mathrm{P}^{+}$ & 373.0 & -2.5 & 406.5 & -17.6 & 347.1 & -14.6 \\
\hline $\mathrm{Me}_{3} \mathrm{~N}^{+}$ & 379.7 & -9.2 & 404.8 & -15.9 & 355.1 & -22.6 \\
\hline $\mathrm{s}^{+}$ & 383.5 & -13.0 & 400.2 & -11.3 & 345.4 & -13.0 \\
\hline \multirow[t]{2}{*}{ Substituent } & \multicolumn{2}{|c|}{$\mathrm{ArNH}_{2}$} & \multicolumn{2}{|c|}{ ArNHCOMe } & \multicolumn{2}{|c|}{$\begin{array}{c}\operatorname{ArC}(\mathrm{Me})= \\
\mathrm{NNHCONH}_{2}\end{array}$} \\
\hline & $\mathrm{BDE}$ & RSE & $\mathrm{BDE}$ & RSE & $\mathrm{BDE}$ & RSE \\
\hline 4-OMe & 378.2 & 8.0 & 400.1 & 15.4 & 381.0 & 3.2 \\
\hline 4-Me & 384.9 & 1.3 & 408.0 & 7.5 & 382.6 & 1.6 \\
\hline $\mathrm{H}$ & 386.2 & (0) & 415.5 & (0) & 384.2 & $(0)$ \\
\hline $4-\mathrm{CN}$ & 398.3 & -12.1 & 424.8 & -9.3 & 388.2 & -4.0 \\
\hline $4-\mathrm{NO}_{2}$ & 404.6 & -18.4 & 427.5 & -12.0 & 387.2 & -3.0 \\
\hline \multirow{2}{*}{ Substituent } & \multicolumn{2}{|c|}{ ArNHPh } & \multicolumn{2}{|c|}{$\mathrm{ArNHNH}_{2}$} & \multicolumn{2}{|c|}{ ArNHOH } \\
\hline & BDE & RSE & BDE & RSE & $\mathrm{BDE}$ & RSE \\
\hline 4-Me & 365 & 2 & 302 & 3 & 284 & 8 \\
\hline $\mathrm{H}$ & 367 & (0) & 305 & (0) & 292 & (0) \\
\hline $4-\mathrm{CN}$ & - & - & 326 & -21 & - & - \\
\hline
\end{tabular}

\begin{tabular}{lcccc}
\hline \hline \multirow{2}{*}{ Substituent } & \multicolumn{2}{l}{$\operatorname{ArNHPO}(\mathrm{OEt})_{2}$} & & \multicolumn{2}{l}{$\operatorname{ArNH}\left(\mathrm{P}^{+} \mathrm{Ph}_{3}\right)$} \\
\cline { 2 - 5 } \cline { 5 - 5 } & $\mathrm{BDE}$ & $\mathrm{RSE}$ & $\mathrm{BDE}$ & $\mathrm{RSE}$ \\
\hline $4-\mathrm{Me}$ & 391 & 6 & 288 & -2 \\
$\mathrm{H}$ & 397 & $(0)$ & 286 & $(0)$ \\
$4-\mathrm{CN}$ & 414 & -17 & 271 & 15 \\
\hline${ }^{a} \mathrm{RSE}=-\Delta \mathrm{BDE}=-\left[\mathrm{BDE}\left(4-\mathrm{G}_{-} \mathrm{C}_{6} \mathrm{H}_{4} \mathrm{R} \bullet\right)-\mathrm{BDE}\left(\mathrm{C}_{6} \mathrm{H}_{5} \mathrm{R} \bullet\right)\right](\mathrm{kJ} / \mathrm{mol})$.
\end{tabular}

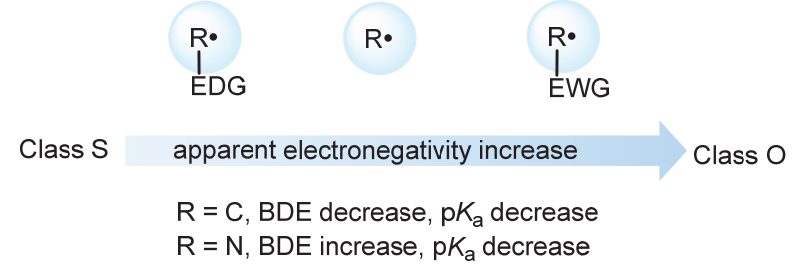

图 4 碳自由基和杂原子自由基的热力学规律

Figure 4 Thermodynamic schedule of carbon and heteroatomic radicals

电子基的自由基, 其中心表观电负性降低, Class $\mathrm{S}$ 性质 更明显.

\section{2 稳态自由基效应}

长期以来, 人们习惯用 “稳定” (stable) 一词来描述 自由基的稳定性，如三苯甲基自由基被认为是一种稳定
的自由基 ${ }^{[1]}$, 不易于进行氢原子转移和对不饱和键加成 等反应，在无氧条件下可以在溶液中稳定存在. 与之相 对应的是甲基自由基等简单烷基自由基，其反应活性很 高，会快速地与其他基团进行自由基反应. 这种定性的 描述比较符合人们的直观认识, 这种判断本质上是基于 对自由基前体的 $\mathrm{BDE}$ 的比较, $\mathrm{BDE}$ 小的分子, 生成的自 由基稳定性高. 但是随后的研究发现 ${ }^{[29]}$, 一些 BDE 较 大的自由基，如三叔丁甲基自由基、2,4,6-三叔丁基苯基 自由基等却表现出传统 “稳定” 自由基的性质. 为了理 解这一问题, 1976 年, Ingold 等 ${ }^{[30]}$ 用 “稳态” (persistent) 一词来描述自由基的稳定性(图 5). Ingold 认为, “稳定” 是通过 BDE 来比较自由基的热力学的相对稳定性, “稳 态” 是通过对自由基寿命的比较来描述自由基的动力学 稳定性. Ingold 将那些显著高于甲基自由基寿命的自由 基称为稳态自由基(persistent radical),一般认为, 自由 基寿命大于 $10^{-3} \mathrm{~s}$ 为稳态自由基，反之则为瞬态自由基 (transient radical). 图 5 列出了一些有代表性的稳态碳自 由基, 位阻效应带来了不利的动力学因素, 因此它们都 具有较长的自由基寿命.

作为高活性物种, 自由基之间的偶联在热力学上是 一个十分有利的过程. 瞬态自由基之间的偶联速率几乎 都是扩散控制的, 反应结果基本上是按照统计学概率进 行的, 呈现出较差的选择性. 但是陆续发现的一系列高 选择性的自由基交叉偶联反应引起了人们的关注，如五 苯基乙烷热解 ${ }^{[31}$ 和一些烷基金属的光解研究 ${ }^{[32]}$ 显示, 体系中高活性的瞬态自由基自偶联是不利的过程(图 6). 研究发现，这些高选择性的反应都是在稳态自由基与瞬 态自由基之间发生的. Fischer 等 ${ }^{[33]}$ 认为该过程中由于稳 态和瞬态自由基以相同的速率生成，随着反应过程中稳 态自由基的累积, 即稳态自由基浓度逐步大于瞬态自由 基浓度, 引导反应生成特定的交叉偶联产物，作者将其 称为 “内生快反应压制” (internal suppression of fast reactions). 1992 年, Finke 等 ${ }^{[32]}$ 提出了稳态自由基效应 (persistent radical effect, PRE): 体系中如果同时存在稳 态自由基和瞬态自由基, 且二者产生速率一致, 那么自 由基交叉偶联将是主产物. 随后的研究显示, 如果体系 中两种瞬态自由基寿命有显著差别, 其稳态自由基效应 也很明显, 即此时得到的主产物是交叉偶联产物 [34].

要实现稳态自由基效应，需满足两个条件：一是交 叉偶联的自由基在体系中产生速率一致，二是两种自由 基的寿命的差别尽可能大. 近年来, 稳态自由基效应在 合成化学中得到了较多的应用 ${ }^{[35]}$, 人们开发了一系列 的有机一有机自由基和有机一金属自由基间交叉偶联反 应，比较有代表性的有机稳态自由基有 TEMPO 、 $t$ - $\mathrm{BuOO}$ ・、氮杂烯丙基自由基和氧芳基阴离子自由基等. 


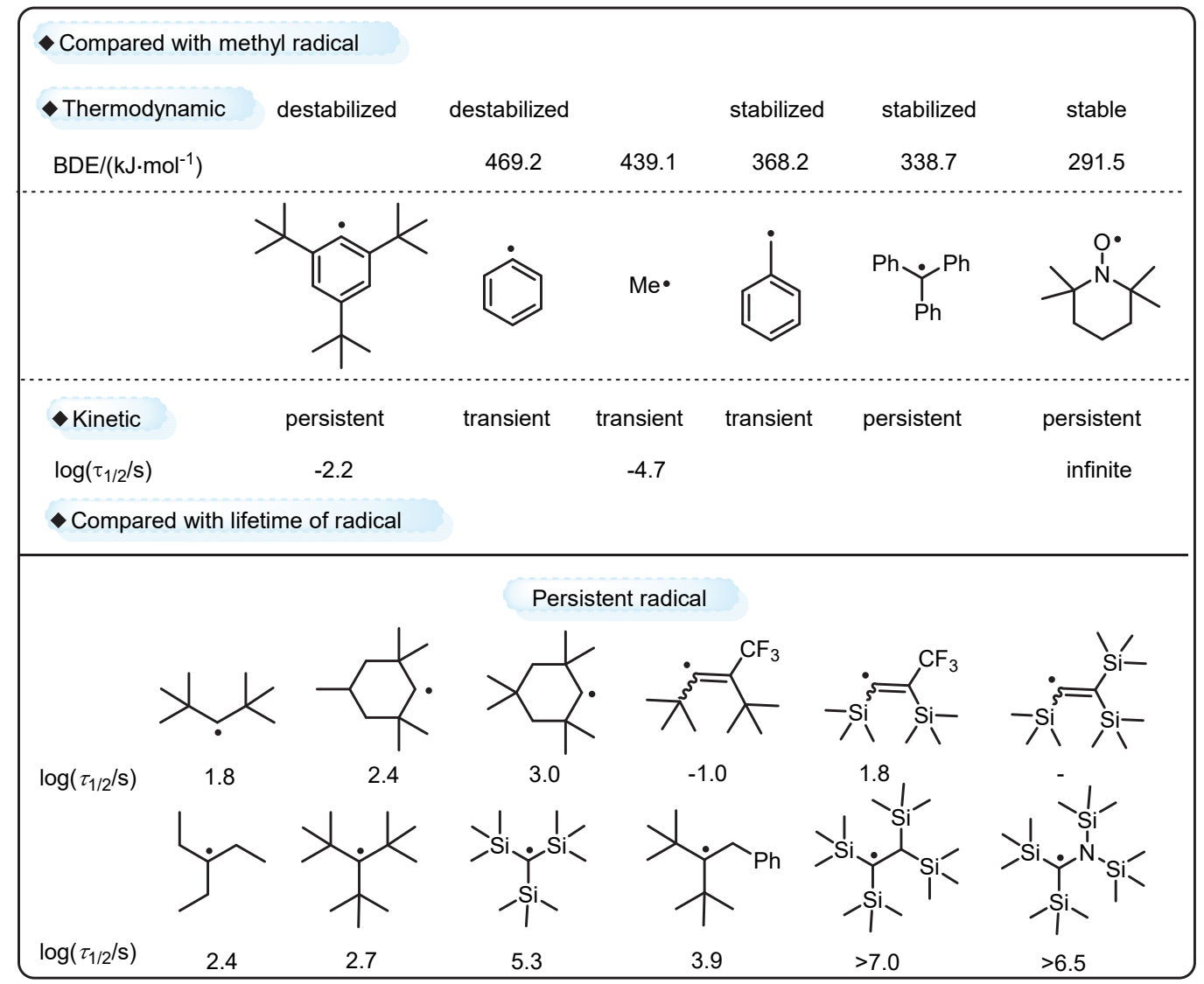

图 5 稳态碳自由基和瞬态碳自由基

Figure 5 Persistent and transient carbon-centered radicals

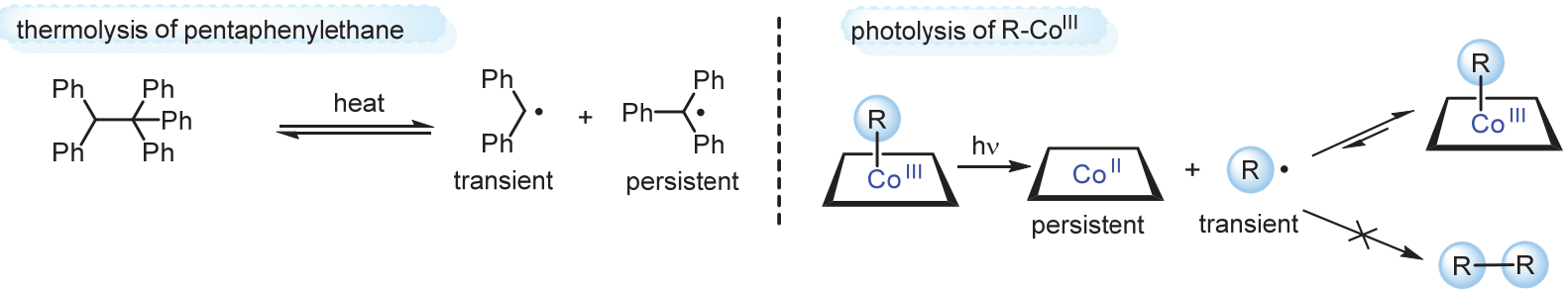

图 6 基于稳态碳自由基效应的反应

Figure 6 PRE-mediated cross-coupling reactions

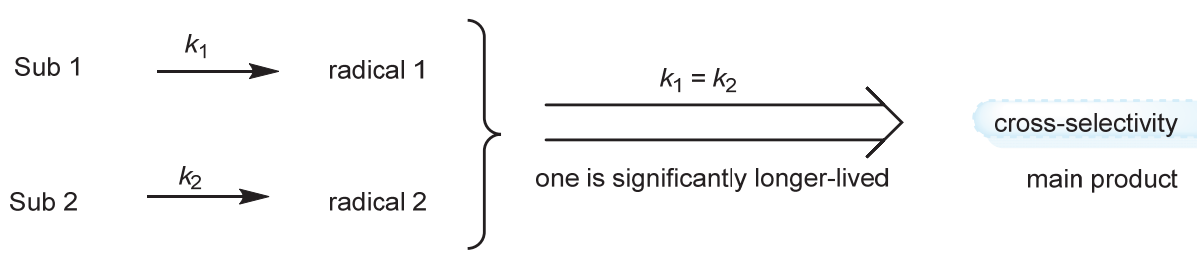

图 7 稳态碳自由基效应

Figure 7 Persistent radical effect

需要说明的是, 这些自由基不一定是严格意义上的稳态 自由基, 但是由于与反应体系中另一种自由基有足够大 的寿命差别, 进而展现出较明显的稳态自由基效应. 除 了有机自由基，许多过渡金属也可以充当稳态自由基角 色，如 $\mathrm{Cr}^{\mathrm{II}[36]} 、 \mathrm{Co}^{\mathrm{II}[37]} 、 \mathrm{Fe}^{\mathrm{II}[38]} 、 \mathrm{Ni}^{\mathrm{II}[39]} 、 \mathrm{Cu}^{\mathrm{II}[40]} 、 \mathrm{Pd}^{\mathrm{II}[41]}$ 、
$\mathrm{Pt}^{\mathrm{I}}[42]$ 等.

\section{3 基于自由基反应动力学的取代基参数}

1937 年, Hammett 等 ${ }^{[43]}$ 以苯甲酸解离为标准反应, 通过线性自由能相关方程(Eq. 4)的建立，首次定义了取 
代基常数 $\sigma$, 这也是定量构效关系研究的里程碑式工作. 取代基常数 $\sigma$ 代表相关取代基对该标准反应影响程度的 大小, 是取代基极性效应和共轭效应的总体反映. 一般 来说, $\sigma$ 常数能够较好模拟与苯甲酸解离过程相近的极 性反应中的取代基效应, 但对反应机理相差过大的其他 类型的反应, 则可能失效. 为更有针对性地反映不同于 苯甲酸解离的其他极性反应中取代基的电子效应，人们 还建立了不同的标准反应, 发展了 $\sigma^{+} 、 \sigma^{-}$等适用于不同 场景极性反应的取代基常数 ${ }^{[44]}$.

对于自由基反应来说, 取代基可以通过自旋离域作 用分散反应中心自由基的电子自旋密度, 从而对反应进 程产生重要影响. 但前述取代基常数是建立在极性反应 的基础上, 其数值并不包含自旋离域效应的贡献, 因此 对于多数自由基反应来说, 极性参数并不能准确地描述 其中的取代基效应. 于是, 人们参考极性参数的建立方 法, 利用一些自由基反应作为标准模式反应来测定相应 的取代基常数.

$$
\lg \left(k / k_{0}\right)=\rho \sigma
$$

早在 1947 年, Alfrey 和 Price ${ }^{[45]}$ 就提出了 $Q-e$ 方程 (Eq. 5), 用于预测烯烃自由基共聚反应的速率, 这也是 量化自由基取代基效应的最初尝试. 其中, $k_{12}$ 表示聚合 物链的末端是 $\mathrm{M}_{1}$ 单体对应的自由基时, 对 $\mathrm{M}_{2}$ 单体进行 加成反应的速率常数, $P_{1}$ 代表自由基链的反应活性, $Q_{2}$ 代表 $\mathrm{M}_{2}$ 单体的反应活性，二者与共轭效应相关， $e_{1} 、 e_{2}$ 分别代表两种单体的极性参数. 定义苯乙烯的 $Q=1$, $e=-0.8$, 即可通过竞聚率实验值 $r_{1}$ 或 $r_{2}$ 计算出其他聚 合单体的 $Q 、 e$ 数值(Eq. 6). 尽管 $Q-e$ 方程只是一个半定 量方法, $Q$ 值也仅能笼统地描述自由基物种整体的共轭 性质而非某一取代基, 但其广泛的适用性与便捷性使其 成为目前最通用的共聚活性方程.

$$
\begin{aligned}
& k_{12}=P_{1} Q_{2} \exp \left(-e_{1} e_{2}\right) \\
& r_{1}=k_{11} / k_{12}=Q_{1} / Q_{2} \exp \left[-e_{1}\left(e_{1}-e_{2}\right)\right]
\end{aligned}
$$

随后, Simamura ${ }^{[46]} 、$ Yamamoto $^{[47]} 、$ Sakurai $^{[48]}$ 、 Kieboom $^{[49]} 、$ Fisher $^{[50]} 、$ Creary ${ }^{[51]} 、 J^{2}$ ackson ${ }^{[52]}$ 等通过对 不同自由基反应速率常数的测定, 分别定义了 $\tau_{\mathrm{p}} 、 E_{\mathrm{R}}$ 、 $E_{\mathrm{D}} 、 E_{\mathrm{R}}{ }^{\mathrm{N}} 、 \sigma_{\mathrm{F}} 、 \sigma_{\mathrm{C}} 、 \sigma_{\mathrm{J}}$. 等取代基常数(图 8), 采用的方法 大多是将自旋离域效应视为影响反应速率的额外因素, 假定间位取代基的自旋离域效应可以忽略, 通过对基于 极性参数的 Hammett 方程进行差额补正, 来获得相应取 代基常数. Arnold 等 ${ }^{[22 b]}$ 也通过电子自旋共振(ESR)实验, 使用自由基对应的超精细耦合常数定义了 $\sigma_{\alpha}$ 常数. 上 述取代基常数已汇总于表 2 , 这些常数一定程度上揭示 了取代基对自由基离域能力的规律, 对于理解自由基性
质和推测反应机理具有重要参考价值.

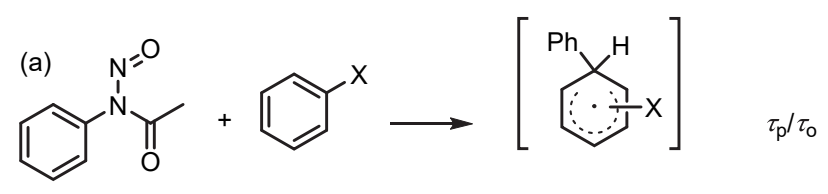

(b)

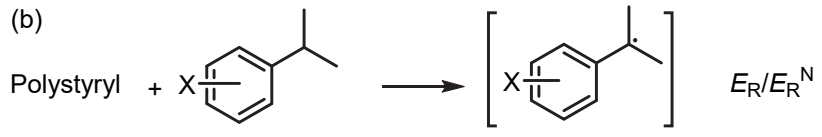

(c)<smiles>[X]c1ccc(C=CC)cc1</smiles>

(d)

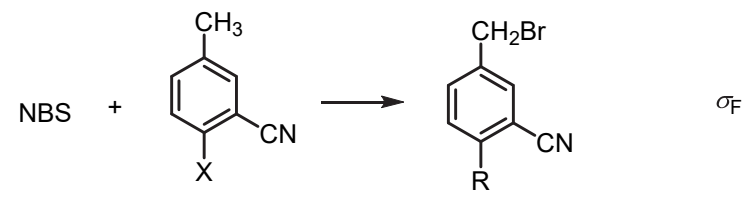

(e)

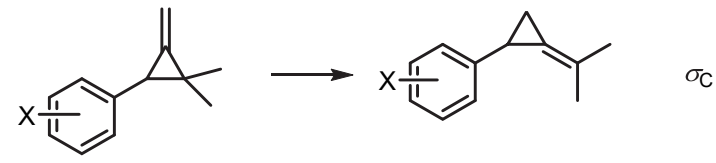

(f)

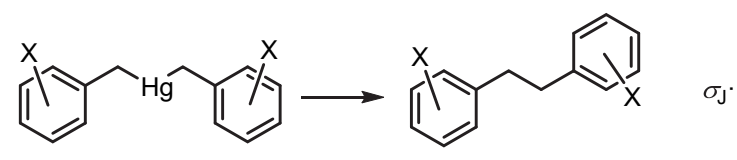

图 8 自由基取代基常数测定的模板反应

Figure 8 Model reactions for determining radical substituent constants

1984 年, 蒋锡咅等 ${ }^{[33]}$ 指出, 上述取代基常数在测定 过程中忽略了取代基极性对苯环 $\pi$ 电子云密度的影响, 而自旋离域作用是与其密切相关的, 所以前述工作并没 有将极性效应和自旋离域效应完全分离开. 作者在考察 三氟苯乙烯热聚反应的过程中发现, 该反应经历了 1,4双自由基的过渡态，而在过渡态中，双自由基的对称性 使其偶极不再发生变化，也不产生新的极性相互作用， 因此额外的加速效应完全由自旋离域作用产生，可以实 现极性效应和自旋离域效应的拆分(图 9). 根据三氟苯 乙烯的结构特性, 作者将 $\mathrm{F}^{1}$ 和 $\mathrm{F}^{3}$ 的氟谱化学位移差值 与取代基极性效应很好地关联起来, 定义了适用于该类 化合物的极性取代基常数 $\sigma_{\mathrm{mb}}{ }^{[54]}$ (Eq. 7). 在 SwainLupton 双参数自由能相关模型的启发下 ${ }^{[55]}$, 作者假设 间位甲基的自旋离域效应为零, 规定反应常数 $\rho \bullet=1$, $\rho_{\mathrm{mb}}=-0.35$, 根据实验测定的动力学数据, 利用二元线 性回归法得到了一系列不同取代基的 $\sigma_{\mathrm{JJ}}$. 值 ${ }^{[22 \mathrm{a}]}$ (Eq. 8, 表 3). 
表 2 自由基取代基常数汇总表

Table 2 Compilation for radical substituent constants

\begin{tabular}{|c|c|c|c|c|c|c|c|c|}
\hline Substituent & $\tau_{\mathrm{p}}$ & $E_{\mathrm{R}}$ & $E_{\mathrm{D}}$ & $E_{\mathrm{R}}{ }^{\mathrm{N}}$ & $\sigma_{\mathrm{F}} \cdot$ & $\sigma_{\mathrm{C}}$ & $\sigma_{\mathrm{J}}$ & $\sigma_{\alpha}$ \\
\hline $\mathrm{H}$ & 0 & 0 & 0 & 0 & 0 & 0 & 0 & 0 \\
\hline$p-\mathrm{NO}_{2}$ & 0.90 & 0.41 & 0.27 & 0.41 & 0.27 & & 0.76 & \\
\hline$p-\mathrm{N}=\mathrm{NPh}$ & 0.90 & & & & 0.33 & & & \\
\hline$p-\mathrm{NMe}_{2}$ & & 0.24 & & -0.11 & & & & \\
\hline$p-\mathrm{Ph}$ & & & & & & & 0.42 & \\
\hline$p$-COMe & & 0.24 & & 0.24 & 0.12 & & & 0.066 \\
\hline$p-\mathrm{COPh}$ & & & & & & & & 0.064 \\
\hline$p-\mathrm{CN}$ & & 0.24 & 0.32 & 0.23 & 0.53 & 0.46 & & 0.043 \\
\hline$p$-SMe & & & & & 0.34 & 0.43 & & \\
\hline$p-\mathrm{CO}_{2} \mathrm{Me}$ & & & & & & 0.35 & & 0.048 \\
\hline$p$-SOMe & & & & & & & & 0.006 \\
\hline$p-\mathrm{SO}_{3} \mathrm{Me}$ & & & & & & & & 0.003 \\
\hline$p$-OCOMe & & & & & & & & 0.001 \\
\hline$p$-OMe & 0.14 & 0.11 & 0.19 & -0.008 & -0.12 & 0.24 & 0.42 & 0.034 \\
\hline$p-\mathrm{SiMe}_{3}$ & & & & & & 0.17 & & \\
\hline$p-\mathrm{Br}$ & & 0.12 & & 0.072 & 0.17 & 0.14 & & \\
\hline$p-{ }^{t} \mathrm{Bu}$ & & 0.03 & & 0.014 & & 0.13 & & 0.036 \\
\hline$p-{ }^{i} \operatorname{Pr}$ & & 0.03 & & 0.034 & & & & \\
\hline$p-\mathrm{F}$ & & & & & & & & -0.011 \\
\hline$p-\mathrm{Cl}$ & 0.16 & 0.10 & 0.07 & 0.062 & 0.08 & 0.12 & 0.18 & 0.017 \\
\hline$p$-I & & 0.12 & & 0.037 & 0.16 & & & \\
\hline$p$-OPh & & 0.13 & & -0.147 & & & & \\
\hline$p$-Me & 0.09 & 0.03 & 0.11 & -0.020 & -0.02 & 0.11 & 0.39 & 0.015 \\
\hline$p-\mathrm{CF}_{3}$ & & & & & & 0.08 & & 0.001 \\
\hline$m-\mathrm{SiMe}_{3}$ & & & & & & 0.03 & & \\
\hline$m-\mathrm{Me}$ & & & & & & -0.02 & & -0.001 \\
\hline$m-\mathrm{CO}_{2} \mathrm{Me}$ & & & & & & & & -0.004 \\
\hline$m$-OMe & & & & & & -0.02 & & \\
\hline$m-\mathrm{Cl}$ & & & & & & -0.04 & & -0.001 \\
\hline$m-\mathrm{F}$ & & & & & & -0.05 & & \\
\hline$m-\mathrm{CF}_{3}$ & & & & & & -0.07 & & -0.014 \\
\hline$m-\mathrm{F}$ & & & & & & -0.08 & & -0.018 \\
\hline$m-\mathrm{CN}$ & & & & & & -0.12 & & -0.039 \\
\hline$m$-OPh & & & & & & & & -0.013 \\
\hline
\end{tabular}

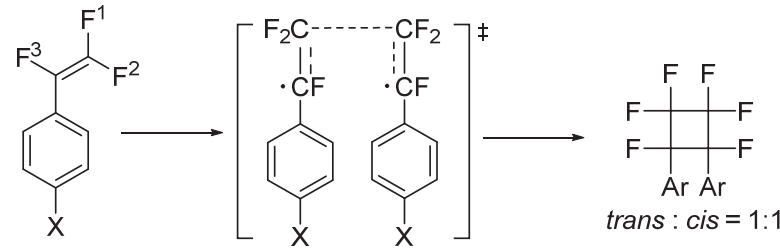

图 9 取代三氟苯乙烯的 $[2+2]$ 反应

Figure $9[2+2]$ reaction of substituted trifluorostyrenes

$$
\begin{aligned}
& \sigma_{\mathrm{mb}}=0.16 \Delta \delta_{3-1}-0.09 \\
& \lg \left(k / k_{0}\right)=\rho_{\mathrm{mb}} \sigma_{\mathrm{mb}}+\rho . \sigma_{\mathrm{JJ}} .
\end{aligned}
$$

随后，蒋锡銺等 ${ }^{[56]}$ 展示了 $\sigma_{\mathrm{JJ}}$. 常数的实用性. 以取 代 $\alpha$-甲基苯乙烯的双官能化反应为例 ${ }^{[57]}$ (图 10), 反应经 历自由基加成，生成溴甲基取代的苄基自由基，随后该 自由基被氧气捕获生成产物. 通过对反应速率常数比、 $\sigma_{\mathrm{mb}}$ 与 $\sigma_{\mathrm{JJ}}$. 进行二元线性回归分析, 可以求得 $\rho_{\mathrm{mb}}=$ -0.168 和 $\rho_{\mathrm{JJ}}=0.449$, 且相关性很好 $(R=0.997)$; 而若 用极性参数 $\sigma^{+}$进行一元回归分析则相关性较差 $(R=$ 0.743). 从反应常数的数值可以看出, 极性和自旋离域 效应对该反应都有较大影响.

$\sigma_{\mathrm{mb}}$ 与 $\sigma_{\mathrm{JJ}}$ 数值已汇总于表 3 . 绝大多数的取代基常 数为正值, 说明取代基普遍起到稳定自由基的作用，而 作者在对自由基反应的测试中发现，所有的反应常数 $\rho_{\mathrm{JJ}}$ 都大于 0 , 说明在反应过渡态中, 取代基均能通过自 旋离域效应，一定程度上分散反应中心的自旋密度分 布. 值得一提的是, 蒋锡㖜等提出的 $\sigma_{\mathrm{JJ}}$. 常数是目前为 止利用动力学方法描述 Class $\mathrm{S}$ 自由基反应活性的精度 最高、体系最全、适用范围最广的一组自由基取代基常 数. 


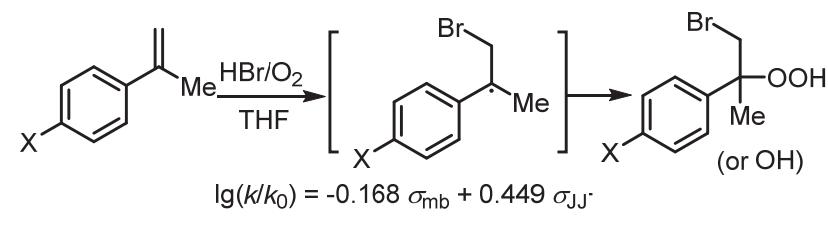

图 $10 \alpha$-甲基苯乙烯的自由基加成反应

Figure 10 Radical addition of $\alpha$-methylstryene

\section{4 自由基的亲核性与亲电性}

早在 20 世纪上半叶, 人们就注意到, 尽管自由基通 常是电中性的物种, 其反应活性和选择性仍会受到极性 效应的影响 ${ }^{[58]}$. 参考非自由基物种亲核性与亲电性的 区分方法，人们将易与缺电子物质发生反应的自由基称 为亲核自由基，易与富电子物质发生反应的自由基称为 亲电自由基, 遵循这种极性匹配原则的反应在动力学上 更有利. Roberts ${ }^{[59]}$ 利用氢原子转移反应过渡态的电子偏 向来定义自由基的亲核性与亲电性(图 11a), 如果 TS-3 的贡献较大, 则 $\mathrm{A}$ 为亲电自由基, $\mathrm{B}$ 为亲核自由基; 如果 TS-4 的贡献较大, 则 $\mathrm{A}$ 为亲核自由基, $\mathrm{B}$ 为亲电自由基.

根据我们对自由基极性的理解，总结了一个基于单 电子转移过程快速判断自由基亲核性与亲电性的方法. 自由基得失电子后分别形成相应的负离子和正离子. 如 果正离子更稳定, 那么该自由基倾向于失去电子, 表现 亲核性; 如果负离子更稳定, 那么该自由基倾向于得到 电子, 表现出亲电性. 以环戊二烯自由基和环庚三烯自 由基为例(图 11b). 环戊二烯自由基得到电子形成具有 芳香性的环戊二烯负离子, 更加稳定, 因此环戊二 (a) hydrogen atom transfer

$$
\begin{aligned}
& \mathrm{A} \cdot+\mathrm{H}-\mathrm{B} \longrightarrow \mathrm{A}-\mathrm{H}+\mathrm{B} \\
& {\left[\mathrm{A} \cdot \mathrm{H}-\left.\mathrm{B}\right|^{\ddagger} \longleftrightarrow \mid \mathrm{A}-\mathrm{H} \mathrm{B}^{\cdot}\right]^{\ddagger} \longleftrightarrow\left[\mathrm{A}^{-} \mathrm{H}^{\cdot} \cdot \mathrm{B}^{+}\right]^{\ddagger} \longleftrightarrow\left|\mathrm{A}^{+} \mathrm{H}^{\cdot} \cdot \mathrm{B}^{-}\right|^{\ddagger}} \\
& \text { TS-1 TS-2 } \quad \text { TS-3 } \\
& \text { TS-4 }
\end{aligned}
$$

(b) single electron transfer

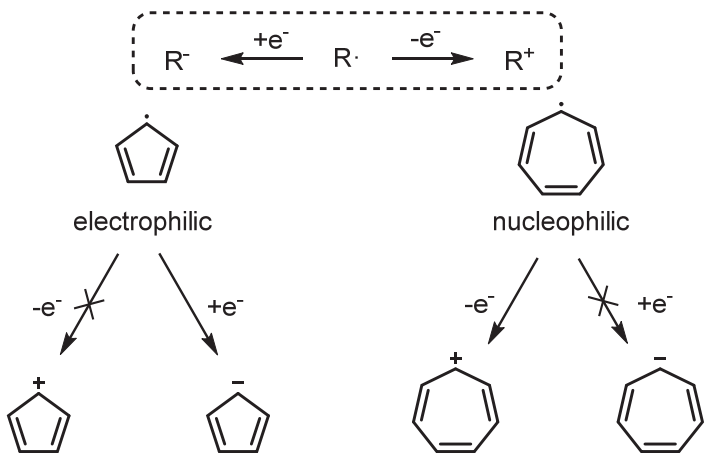

图 11 亲核性与亲电性自由基的定性判据

Figure 11 Qualitative criteria for nucleophilic and electrophilic radicals

烯自由基为亲电自由基; 类似地，环庚三烯自由基失去 电子形成具有芳香性的环庚三烯正离子，更加稳定，因 此环庚三烯自由基为亲核自由基.

早期关于自由基亲核性与亲电性的研究比较碎片 化、定性化，主要从单一反应入手，通过比较反应速率、 活化能等参数来对比自由基的极性差别, 并以此进行亲 核性与亲电性的划分. 这种区分方式由于标准不统一, 所得结果往往争议较大。以氰基甲基自由基为例

\begin{tabular}{|c|c|c|c|c|c|}
\hline Substituent & $\sigma_{\mathrm{mb}}$ & $\sigma_{\mathrm{JJ}}$ & Substituent & $\sigma_{\mathrm{mb}}$ & $\sigma_{\mathrm{JJ}}$ \\
\hline $\mathrm{H}$ & 0 & 0 & $p$-OMe & -0.77 & 0.23 \\
\hline$p-\mathrm{NMe}_{2}$ & -0.96 & 1.00 & $p-\mathrm{Br}$ & 0.13 & 0.23 \\
\hline$p-\mathrm{CF}=\mathrm{CF}_{2}$ & 0.40 & 0.86 & $p-\mathrm{Cl}$ & 0.11 & 0.22 \\
\hline$p-\mathrm{SMe}$ & -0.18 & 0.62 & $p-\mathrm{CH}=\mathrm{CH}_{2}$ & 0.03 & \\
\hline$p$-COMe & 0.56 & 0.54 & $p$-Me & -0.29 & 0.15 \\
\hline$p$-SOMe & 0.19 & 0.50 & $p$-Et & & 0.15 \\
\hline$p-\mathrm{Ph}$ & 0.06 & 0.47 & $p-\mathrm{CF}_{3}$ & 0.49 & -0.01 \\
\hline$p-\mathrm{CN}$ & 0.86 & 0.42 & $p-\mathrm{F}$ & -0.24 & -0.02 \\
\hline$p-\mathrm{COOH}$ & 0.31 & 0.38 & $m-\mathrm{Me}$ & -0.20 & 0 \\
\hline$p-\mathrm{CONH}_{2}$ & 0.10 & 0.38 & $m-\mathrm{Br}$ & 0.36 & 0.12 \\
\hline$p-\mathrm{SO}_{2} \mathrm{Me}$ & 0.64 & 0.41 & $m-{ }^{t} \mathrm{Bu}$ & -0.20 & 0.11 \\
\hline$p-\mathrm{NO}_{2}$ & 0.86 & 0.36 & $m-\mathrm{CN}$ & 0.89 & 0.11 \\
\hline$p$-OCOMe & -0.14 & 0.35 & $m-\mathrm{OMe}$ & -0.11 & 0.10 \\
\hline$p-\mathrm{CO}_{2} \mathrm{Me}$ & 0.48 & 0.33 & $m-\mathrm{CO}_{2} \mathrm{Me}$ & 0.21 & 0.10 \\
\hline$p-\mathrm{SO}_{2} \mathrm{Me}$ & 0.64 & 0.41 & $m$-Et & -0.15 & \\
\hline$p-\mathrm{SiMe}_{3}$ & 0.14 & 0.31 & $m-\mathrm{F}$ & 0.23 & 0.03 \\
\hline$p-{ }^{c} \operatorname{Pr}$ & -0.31 & 0.29 & $m-\mathrm{NO}_{2}$ & 0.69 & 0.001 \\
\hline$p-{ }^{t} \mathrm{Bu}$ & -0.22 & 0.26 & $m-\mathrm{Cl}$ & 0.12 & -0.05 \\
\hline$p$-OPh & -0.46 & & $m-\mathrm{CF}_{3}$ & 0.39 & -0.07 \\
\hline
\end{tabular}
$\left(\cdot \mathrm{CH}_{2} \mathrm{CN}\right)$, Radom 等 ${ }^{[60]}$ 使用高级量子化学从头计算法研

表 $3 \sigma_{\mathrm{mb}}$ 与 $\sigma \mathrm{J}$ 。值

Table $3 \sigma_{\mathrm{mb}}$ and $\sigma_{\mathrm{JJ}} \cdot$ values 
究了一系列自由基加成反应, 发现 $\cdot \mathrm{CH}_{2} \mathrm{CN}$ 表现出明显 的亲电自由基性质, Fischer 等[61]在时间分辨电子自旋共 振(Time-resolved ESR)研究中认为, $\cdot \mathrm{CH}_{2} \mathrm{CN}$ 具有弱亲电 性, 而 Giese 等 ${ }^{[62]}$ 通过自由基加成反应速率实验值的研 究, 认为 $\cdot \mathrm{CH}_{2} \mathrm{CN}$ 的极性处于亲核和亲电自由基的边界.

自由基的亲核性与亲电性标度由于受反应物、反应 条件等多重因素影响, 实验测定难度较大, 难以有效反 映其本征属性. 1998 年, Héberger 和 Lopata 等 ${ }^{[63]}$ 使用主 成分分析法(Principal Component Analysis)对影响自由 基加成反应的因素做了拆分, 基于自由基加成反应的实 验数据和计算数据, 作者将自由基的极性与三个热力学 描述符进行相关, 即烯烃的电子亲和势(EA)、电离电势 (一IP)以及反应放热值 $\left(-\Delta H_{\mathrm{r}}\right)$, 并以此进行自由基的分 类. 这也是对自由基极性首次较为系统的分类，但该方 法建立的只是半定量的相对标度.

2007 年, De Proft 等[64]参考非自由基物种亲电性指 数的建立方法, 定义了自由基全局亲电性指数 $\omega$ (Eq. 9) 以及对自由基中心的局部亲电性指数 $\omega^{+}{ }_{\mathrm{rc}}{ }^{[65]}$ (Eq. 10), 其中 $\mu$ 为电子化学势, $\eta$ 是化学硬度, 可以通过垂直电离 能(IP)和电子亲和势(EA)计算得到(Eq. 11), $f^{+}(\mathrm{r})$ 为亲核 进攻的 Fukui 函数. 对于亲核性指数, 作者参考 Jaramillo 等[66]提出的经验模型定义了全局亲核性指数
$\omega^{-}$(Eq. 12), 其中 $\mathrm{A}$ 代表亲核试剂, B 代表亲电试剂，以 上数据均可以通过理论计算得到. 作者考察了 35 种常 见自由基的亲核性与亲电性，并对其做出分类，其结果 与上述主成分分析法所得结果相近(图 12). 该方法建立 了自由基极性的绝对标度, 并且不依赖反应参数, 提供 了一个方便快捷的自由基极性比对方法. 但由于该方法 在计算过程中只考虑单个独立分子，反映气相状态下的 极性指数，在实际应用中参考性不足，De Proft 等 ${ }^{[67]}$ 随 后在自由基亲电性指数的计算中加入反映溶剂效应的 因素. 作者使用 COSMO 和 IEF-PCM 溶剂化模型进行修 正，对正己烷、二氯甲烷、异丙醇、丙酮和水五种溶剂 下的亲电性指数进行了计算, 结果表明自由基的化学势 $\mu$ 在溶液中变化不大, 而化学硬度 $\eta$ 大幅度降低, 因此 溶液中自由基的亲电性指数整体高于气相.

$$
\begin{aligned}
& \omega=\mu^{2} / 2 \eta \\
& \omega_{\mathrm{rc}}^{+}=\omega f^{\dagger}(r) \\
& \mu=-(\mathrm{IP}+\mathrm{EA}) / 2 \quad \eta=\mathrm{IP}-\mathrm{EA} \\
& \omega^{-}=\frac{\left(\mu_{\mathrm{A}}-\mu_{\mathrm{B}}\right)^{2}}{2\left(\eta_{\mathrm{A}}+\eta_{\mathrm{B}}\right)^{2}} \eta_{\mathrm{A}}
\end{aligned}
$$

图 12 自由基的 $\omega$ 指数

Figure $12 \omega$ index of radicals 
2013 年, Domingo 等 ${ }^{[68]}$ 使用类似的方法建立了全局 亲电性指数 $\omega^{\mathrm{o}}$ 、局部亲电性指数 $\omega^{\mathrm{o}} \mathrm{k}$, 作者也基于 $\mathrm{HOMO}$ 轨道能量对全局亲核性指数 $N^{\circ}$ 、局部亲核性指 数 $N_{\mathrm{k}}^{\mathrm{o}}$ 的定义做出了修改, 测试范围也扩大到一些复杂 结构的自由基, 如多取代烷基、烯丙基等. 随后, 吕东梅 等 ${ }^{[69]}$ 使用 Domingo 建立的标度, 对大量硼自由基的亲 核性与亲电性进行了考察. 研究表明硼自由基的极性范 围比碳自由基更宽, 因此也显示硼自由基具有潜在更丰 富的反应活性.

2017 年, Santschi 等[70]利用实验方法对亲电性指数 进行了测定. 作者以自由基对组氨酰胺的加成为模板反 应(图 13), 通过线性自由能相关来定义亲电性指数 $M$ (Eq. 13), 其中 $K$ 为反应平衡常数. 由于组氨酰胺具有较 高的氧化电位, 适合用来研究强氧化性自由基的性质, 加成反应在水相中进行, 通过激光闪光光解的方式来引 发反应. 作者以甲基自由基为参照测定了一系列自由基 的 $M$ 指数(表 4), $M$ 值与 De Proft 等计算得到的 $\omega\left(\mathrm{H}_{2} \mathrm{O}\right)$ 指数有较好的相关性 $\left(R^{2}=0.88\right)$.

$$
M=\log K-\log K_{\mathrm{CH}_{3}}
$$

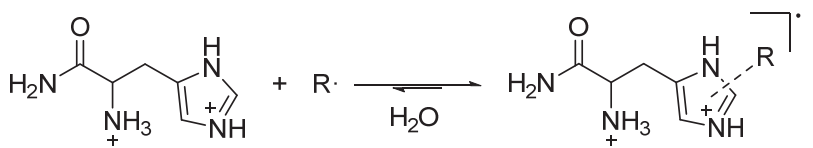

图 13 组氨酰胺自由基加成反应

Figure 13 Radical addition of histamine amide

表 4 自由基的 $M$ 指数

Table $4 \mu$ index for radicals

\begin{tabular}{lll}
\hline Substituent & $\log K$ & $M\left(\mathrm{H}_{2} \mathrm{O}\right)$ \\
\hline$\cdot \mathrm{H}$ & 4.70 & 1.47 \\
$\cdot \mathrm{OH}$ & 4.33 & 1.10 \\
$\cdot \mathrm{CH}_{3}$ & 3.23 & 0.00 \\
$\cdot \mathrm{CF}_{3}$ & 4.18 & 0.95 \\
$\cdot \mathrm{CCl}_{3}$ & 4.23 & 1.00 \\
$\cdot \mathrm{CH}_{2} \mathrm{OH}$ & 2.48 & -0.75 \\
$\cdot \mathrm{CH}_{2} \mathrm{CH}_{2} \mathrm{OH}$ & 2.00 & -1.23 \\
$\left.\cdot \mathrm{C}^{(\mathrm{CH}}\right)_{2} \mathrm{OH}$ & 2.00 & -1.23 \\
$\cdot \mathrm{CH}_{2} \mathrm{C}(\mathrm{OH})\left(\mathrm{CH}_{3}\right)_{2}$ & 3.60 & 0.37 \\
\hline
\end{tabular}

\section{5 总结}

经过百余年的发展, 人们从热力学和动力学角度建 立了一套较完善的衡量自由基影响因素的方法. 通过对 各种取代基常数和寿命的测定, 对自由基的反应活性有 了较全面的认识. 相对于动力学专注于对自由基反应速 率的考察, 通过对自由基前体的键均裂能的比较, 可以 直观地判断自由基的热力学稳定性. 这两种策略是从不 同角度对自由基性质的考察. 这些数据还反映了自由基
的极性性质，通过对极性的定量化，使自由基的反应性 质更加明确，对设计、优化化学反应以及后续的机理研 究都有重要的影响. 但是相对于飞速发展的有机反应方 法学, 自由基的定量化参数仍然比较局限, 适用范围仍 待提高, 某些底物的定量参数难以准确测定, 这些都是 困扰自由基定量参数进一步应用的问题，相信随着检测 技术的进步和方法的改善，衡量自由基性质的参数会更 加完善和精确.

\section{References}

[1] Gomberg, M. J. Am. Chem. Soc. 1900, 22, 757.

[2] (a) Walling, C. Tetrahedron 1985, 41, 3887. (b) Ingold, K. U. Pure Appl. Chem. 1997, 69, 241.

[3] Yan, M.; Lo, J. C.; Edwards, J. T.; Baran, P. S. J. Am. Chem. Soc. 2016, 138, 12692.

[4] (a) Romero, K. J.; Galliher, M. S.; Pratt, D. A.; Stephenson, C. R. J. Chem. Soc. Rev. 2018, 47, 7851.

(b) Shashni, B.; Nagasaki, Y. J. Pers. Med. 2021, 11, 92.

[5] (a) Jeschke, P. ChemBioChem 2004, 5, 571.

(b) Reichel, M.; Karaghiosoff, K. Angew. Chem., Int. Ed. 2020, 59, 12268 .

[6] (a) Ratera, I.; Veciana, J. Chem. Soc. Rev. 2012, 41, 303. (b) Huang, Y.; Egap, E. Polym. J. 2018, 50, 603.

[7] McMillen, D. F.; Golden, D. M. Annu. Rev. Phys. Chem. 1982, 33, 493.

[8] Xue, X.-S.; Ji, P.; Zhou, B.; Cheng, J.-P. Chem. Rev. 2017, 117, 8622 .

[9] (a) Bordwell, F. G.; Bausch, M. J. J. Am. Chem. Soc. 1986, 108, 1979.

(b) Bordwell, F. G.; Bausch, M. J. J. Am. Chem. Soc. 1986, 108, 2473.

(c) Bordwell, F. G.; Cheng, J. P.; Harrelson, J. A. J. Am. Chem. Soc. 1988, 110, 1229.

[10] Bordwell, F. G.; Cheng, J. P.; Bausch, M. J. J. Am. Chem. Soc. 1988, 110, 2872 .

[11] (a) Bordwell, F. G.; Cheng, J. P.; Bausch, M. J. J. Am. Chem. Soc. 1988, 110, 2867.

(b) Bordwell, F. G.; Cheng, J. P.; Seyedrezai, S. E.; Wilson, C. A. J. Am. Chem. Soc. 1988, 110, 8178.

[12] Bordwell, F. G.; Cheng, J. J. Am. Chem. Soc. 1991, 113, 1736.

[13] Bordwell, F. G.; Zhang, X. M.; Cheng, J. P. J. Org. Chem. 1993, 58, 6410 .

[14] Bordwell, F. G.; Zhang, X.; Alnajjar, M. S. J. Am. Chem. Soc. 1992, $114,7623$.

[15] Bordwell, F. G.; Harrelson, J. A.; Lynch, T. Y. J. Org. Chem. 1990 $55,3337$.

[16] (a) Zhang, X. M.; Bordwell, F. G. J. Am. Chem. Soc. 1994, 116, 968.

(b) Bordwell, F. G.; Zhang, X. M. Acc. Chem. Res. 1993, 26, 510.

[17] Yang, J.-D.; Xue, X.-S.; Ji, P.; Li, X.; Cheng, J.-P. Internet Bond-energy Databank ( $p K_{a}$ and BDE): iBonD Home Page, http:// ibond.chem.tsinghua.edu.cn or http://ibond.nankai.edu.cn.

[18] Cheng, J.-P.; Lu, Y.; Liu, B.; Zhao, Y.-X.; Wang, D.-F.; Sun, Y.-K.; Mi, J. -L. Sci. Sin. Chim. 1998, 31, 164 (in Chinese) (程津培, 鲁云, 刘博, 赵永昱, 王涤非, 孙永恺, 米江林, 中国 科学: 化学, 1998, 31, 164.)

[19] Walter, R. I. J. Am. Chem. Soc. 1966, 88, 1923.

[20] (a) Arnett, E. M.; Amarnath, K.; Harvey, N. G.; Cheng, J. J. Am. Chem. Soc. 1990, 112, 344.

(b) Parker, V. D. J. Am. Chem. Soc. 1992, 114, 7458.

(c) Wayner, D. D. M.; Parker, V. D. Acc. Chem. Res. 1993, 26, 287.

[21] (a) Cheng, J. -P.; Zhao, Y.-X.; Hai, Z.-W. Sci. Sin. Chim. 1995, 28, 804 (in Chinese). 
(程津培, 赵永显, 还振威, 中国科学: 化学, 1995, 28, 804.)

(b) Cheng, J.-P. Ph.D. Dissertation, Northwestern University, Evanston, 1987.

[22] (a) Jiang, X.; Ji, G. J. Org. Chem. 1992, 57, 6051.

(b) Dust, J. M.; Arnold, D. R. J. Am. Chem. Soc. 1983, 105, 1221.

[23] (a) Cheng, J.-P.; Liu, B.; Zhao, Y.; Wen, Z.; Sun, Y. J. Am. Chem. Soc. 2000, 122, 9987.

(b) Cheng, J.-P.; Liu, B.; Zhao, Y.; Sun, Y.; Zhang, X.-M.; Lu, Y. J. Org. Chem. 1999, 64, 604.

[24] Cheng, J.-P.; Zhao, Y. Tetrahedron 1993, 49, 5267.

[25] (a) Viehe, H. G.; Janousek, Z.; Merenyi, R.; Stella, L. Acc. Chem. Res. 1985, 18, 148.

(b) Bordwell, F. G.; Lynch, T. Y. J. Am. Chem. Soc. 1989, 111, 7558.

[26] Zhao, Y.-X.; Hai, Z.-W.; Cheng, J.-P. Acta Chim. Sinica 1994, 52, 908 (in Chinese).

(赵永显, 还振威, 程津培, 化学学报, 1994, 52, 908.)

[27] (a) Wen, Z.; Li, Z.; Shang, Z.; Cheng, J.-P. J. Org. Chem. 2001, 66, 1466 .

(b) Song, K.-S.; Liu, L.; Guo, Q.-X. J. Org. Chem. 2003, 68, 4604.

(c) Nam, P.-C.; Nguyen, M. T.; Chandra, A. K. J. Phys. Chem. A 2005, 109, 10342.

(d) Menon, A. S.; Henry, D. J.; Bally, T.; Radom, L. Org. Biomol. Chem. 2011, 9, 3636.

[28] Cheng, J. -P.; Zhao, Y. -Y.; Yuan, Y. -F. Chem. Bull. 1993, 6, 18 (in Chinese).

(程津培，赵永昱，袁耀峰，化学通报, 1993, 6, 18.)

[29] Hicks, R. G. Org. Biomol. Chem. 2007, 5, 1321.

[30] Griller, D.; Ingold, K. U. Acc. Chem. Res. 1976, 9, 13.

[31] Bachmann, W. E.; Wiselogle, F. Y. J. Org. Chem. 1936, 01, 354.

[32] Daikh, B. E.; Finke, R. G. J. Am. Chem. Soc. 1992, 114, 2938.

[33] Fischer, H. J. Am. Chem. Soc. 1986, 108, 3925.

[34] Fischer, H. Chem. Rev. 2001, 101, 3581.

[35] (a) Leifert, D.; Studer, A. Angew. Chem., Int. Ed. 2020, 59, 74.

(b) Studer, A. Chem.-Eur. J. 2001, 7, 1159.

[36] (a) Maciel, G. E.; McIver, J. W.; Ostlund, N. S.; Pople, J. A. J. Am. Chem. Soc. 1970, 92, 1.

(b) Mitsunuma, H.; Tanabe, S.; Fuse, H.; Ohkubo, K.; Kanai, M. Chem. Sci. 2019, 10, 3459.

(c) Schwarz, J. L.; Schäfers, F.; Tlahuext-Aca, A.; Lückemeier, L.; Glorius, F. J. Am. Chem. Soc. 2018, 140, 12705.

[37] (a) Michiyuki, T.; Komeyama, K. Asian J. Org. Chem.2020, 9, 343. (b) Demarteau, J.; Debuigne, A.; Detrembleur, C. Chem. Rev. 2019 , 119, 6906.

(c) Pattenden, G. Chem. Soc. Rev. 1988, 17, 361.

[38] Kyne, S. H.; Lefèvre, G.; Ollivier, C.; Petit, M.; Ramis Cladera, V.-A.; Fensterbank, L. Chem. Soc. Rev. 2020, 49, 8501.

[39] (a) Milligan, J. A.; Phelan, J. P.; Badir, S. O.; Molander, G. A. Angew. Chem., Int. Ed. 2019, 58, 6152.

(b) Gui, Y.-Y.; Sun, L.; Lu, Z.-P.; Yu, D.-G. Org. Chem. Front. 2016, 3, 522 .

(c) Tasker, S. Z.; Standley, E. A.; Jamison, T. F. Nature 2014, 509, 299.

[40] (a) Liu, Z.; Xiao, H.; Zhang, B.; Shen, H.; Zhu, L.; Li, C. Angew. Chem., Int. Ed. 2019, 58, 2510.

(b) Wang, F.; Wang, D.; Zhou, Y.; Liang, L.; Lu, R.; Chen, P.; Lin, Z.; Liu, G. Angew. Chem., Int. Ed. 2018, 57, 7140 .

(c) Wang, F.; Chen, P.; Liu, G. Acc. Chem. Res. 2018, 51, 2036.

[41] (a) Wang, G.-Z.; Shang, R.; Cheng, W.-M.; Fu, Y. J. Am. Chem. Soc. 2017, 139, 18307.

(b) Zhou, W.-J.; Cao, G.-M.; Shen, G.; Zhu, X.-Y.; Gui, Y.-Y.; Ye, J.-H.; Sun, L.; Liao, L.-L.; Li, J.; Yu, D.-G. Angew. Chem., Int. Ed.
2017, 56, 15683

(c) Zhang, H.-H.; Zhao, J.-J.; Yu, S. J. Am. Chem. Soc. 2018, 140, 16914.

(d) Ishiyama, T.; Murata, M.; Suzuki, A.; Miyaura, N. J. Chem. Soc., Chem. Commun. 1995, 295.

(e) Ryu, I.; Kreimerman, S.; Araki, F.; Nishitani, S.; Oderaotoshi, Y.; Minakata, S.; Komatsu, M. J. Am. Chem. Soc. 2002, 124, 3812.

[42] (a) Kratish, Y.; Kostenko, A.; Kaushansky, A.; Tumanskii, B.; Bravo-Zhivotovskii, D.; Apeloig, Y. Angew. Chem., Int. Ed. 2018, 57, 8275 .

(b) Kondo, T.; Tsuji, Y.; Watanabe, Y. Tetrahedron Lett. 1988, 29, 3833 .

(c) Kondo, T.; Sone, Y.; Tsuji, Y.; Watanabe, Y. J. Organomet. Chem. 1994, 473, 163.

[43] Hammett, L. P. J. Am. Chem. Soc. 1937, 59, 96.

[44] Hansch, C.; Leo, A.; Taft, R. W. Chem. Rev. 1991, 91, 165.

[45] Alfrey Jr, T.; Price, C. C. J. Polym. Sci. 1947, 2, 101.

[46] Itô, R.; Migita, T.; Morikawa, N.; Simamura, O. Tetrahedron 1965 21,955 .

[47] Yamamoto, T.; Otsu, T. Chem. Ind. (London) 1967, 787.

[48] Sakurai, H.; Hayashi, S.-I.; Hosomi, A. Bull. Chem. Soc. Jpn. 1971, 44, 1945.

[49] Kieboom, A. P. G. Tetrahedron 1972, 28, 1325

[50] Fisher, T. H.; Meierhoefer, A. W. J. Org. Chem. 1978, 43, 224

[51] (a) Leigh, W. J.; Arnold, D. R.; Humphreys, R. W. R.; Wong, P. C. Can. J. Chem. 1980, 58, 2537.

(b) Creary, X. J. Org. Chem. 1980, 45, 280.

[52] Dinçtürk, S.; Jackson, R. A.; Townson, M.; Ağirbaş, H.; Billingham, N. C.; March, G. J. Chem. Soc., Perkin Trans. 2 1981, 1121.

[53] Jiang, X.-K.; Ji, G.-Z.; Yu, C.-X. Acta Chim. Sinica 1984, 42, 599 (in Chinese). (蒋锡熟，计国桢，于崇䂀，化学学报, 1984, 42, 599.)

[54] Ji, G.-Z.; Jiang, X.-K.; Zhang, Y.-H.; Yuan, S.-G.; Yu, C.-X.; Shi, Y.-Q.; Zhang, X.-L.; Shi, W.-T. J. Phys. Org. Chem. 1990, 3, 643.

[55] (a) Swain, C. G.; Lupton, E. C. J. Am. Chem. Soc. 1968, 90, 4328. (b) Swain, C. G.; Unger, S. H.; Rosenquist, N. R.; Swain, M. S. J. Am. Chem. Soc. 1983, 105, 492.

[56] Jiang, X.-K. Acc. Chem. Res. 1997, 30, 283.

[57] Jiang, X.-K.; Liu, W. W.-Z.; Wu, S.-H. J. Phys. Org. Chem. 1994, 7, 96.

[58] (a) Harris, E. F. P.; Waters, W. A. Nature 1952, 170, 212. (b) Walling, C. Pure Appl. Chem. 1967, 15, 69.

[59] Roberts, B. P. Chem. Soc. Rev. 1999, 28, 25.

[60] Wong, M. W.; Pross, A.; Radom, L. J. Am. Chem. Soc. 1994, 116, 6284.

[61] Wu, J. Q.; Beranek, I.; Fischer, H. Helv. Chim. Acta 1995, 78, 194.

[62] Giese, B.; He, J.; Mehl, W. Chem. Ber. 1988, 121, 2063.

[63] Héberger, K.; Lopata, A. J. Org. Chem. 1998, 63, 8646.

[64] (a) Maynard, A. T.; Huang, M.; Rice, W. G.; Covell, D. G. Proc. Natl. Acad. Sci. U. S. A. 1998, 95, 11578.

(b) Parr, R. G.; Szentpály, L. V.; Liu, S. J. Am. Chem. Soc. 1999, $121,1922$.

[65] De Vleeschouwer, F.; Van Speybroeck, V.; Waroquier, M.; Geerlings, P.; De Proft, F. Org. Lett. 2007, 9, 2721.

[66] Jaramillo, P.; Pérez, P.; Contreras, R.; Tiznado, W.; Fuentealba, P. $J$. Phys. Chem. A 2006, 110, 8181.

[67] De Vleeschouwer, F.; Geerlings, P.; De Proft, F. Theor. Chem. Acc. 2012, 131, 1245 .

[68] Domingo, L. R.; Pérez, P. Org. Biomol. Chem. 2013, 11, 4350.

[69] Wu, C.; Hou, X.; Zheng, Y.; Li, P.; Lu, D. J. Org. Chem. 2017, 82, 2898.

[70] Santschi, N.; Nauser, T. ChemPhysChem 2017, 18, 2973. 